\title{
Level Spacing Distributions and the Bessel Kernel
}

\author{
Craig A. Tracy* \\ Department of Mathematics and Institute of Theoretical Dynamics, \\ University of California, Davis, CA 95616, USA \\ Harold Widom ${ }^{\dagger}$ \\ Department of Mathematics, \\ University of California, Santa Cruz, CA 95064, USA
}

\begin{abstract}
Scaling models of random $N \times N$ hermitian matrices and passing to the limit $N \rightarrow \infty$ leads to integral operators whose Fredholm determinants describe the statistics of the spacing of the eigenvalues of hermitian matrices of large order. For the Gaussian Unitary Ensemble, and for many others as well, the kernel one obtains by scaling in the "bulk" of the spectrum is the "sine kernel" $\frac{\sin \pi(x-y)}{\pi(x-y)}$. Rescaling the GUE at the "edge" of the spectrum leads to the kernel $\frac{\operatorname{Ai}(x) \operatorname{Ai}^{\prime}(y)-\operatorname{Ai}^{\prime}(x) \operatorname{Ai}(y)}{x-y}$ where $\mathrm{Ai}$ is the Airy function. In previous work we found several analogies between properties of this "Airy kernel" and known properties of the sine kernel: a system of partial differential equations associated with the logarithmic differential of the Fredholm determinant when the underlying domain is a union of intervals; a representation of the Fredholm determinant in terms of a Painlevé transcendent in the case of a single interval; and, also in this case, asymptotic expansions for these determinants and related quantities, achieved with the help of a differential operator which commutes with the integral operator. In this paper we show that there are completely analogous properties for a class of kernels which arise when one rescales the Laguerre or Jacobi ensembles at the edge of the spectrum, namely

$$
\frac{J_{\alpha}(\sqrt{x}) \sqrt{y} J_{\alpha}^{\prime}(\sqrt{y})-\sqrt{x} J_{\alpha}^{\prime}(\sqrt{x}) J_{\alpha}(\sqrt{y})}{2(x-y)}
$$

where $J_{\alpha}(z)$ is the Bessel function of order $\alpha$. In the cases $\alpha=\mp \frac{1}{2}$ these become, after a variable change, the kernels which arise when taking scaling limits in the bulk of the spectrum for the Gaussian orthogonal and symplectic ensembles. In particular, an asymptotic expansion we derive will generalize ones found by Dyson for the Fredholm determinants of these kernels.
\end{abstract}

\section{INTRODUCTION AND STATEMENT OF RESULTS}

\section{A. Introduction}

Scaling models of random $N \times N$ hermitian matrices and passing to the limit $N \rightarrow \infty$ leads to integral operators whose Fredholm determinants describe the statistics of the spacing of the eigenvalues of hermitian matrices of large order [18[25]. Which integral operators (or, more precisely, which kernels of integral operators) result depends on the matrix model one starts with and at which location in the spectrum the scaling takes place.

For the simplest model, the Gaussian Unitary Ensemble (GUE), and for many others as well (see, e.g., 16, 17,23,24]), the kernel one obtains by scaling in the "bulk" of the spectrum is the "sine kernel"

$$
\frac{\sin \pi(x-y)}{\pi(x-y)} \text {. }
$$

Precisely, this comes about as follows. If $\left\{\phi_{k}(x)\right\}_{k=0}^{\infty}$ is the sequence obtained by orthonormalizing the sequence $\left\{x^{k} e^{-x^{2} / 2}\right\}$ over $(-\infty, \infty)$ and if

$$
K_{N}(x, y)=\sum_{k=0}^{N-1} \phi_{k}(x) \phi_{k}(y)
$$

*e-mail address: tracy@itd.ucdavis.edu

†e-mail address: widom@cats.ucsc.edu 
then in the GUE the probability density that $n$ of the eigenvalues (irrespective of order) lie in infinitesimal intervals about $x_{1}, \ldots, x_{n}$ is equal to

$$
R_{n}\left(x_{1}, \ldots, x_{n}\right)=\operatorname{det}\left(K_{N}\left(x_{i}, x_{j}\right)\right)_{i, j=1, \ldots, n} .
$$

The density of eigenvalues at a fixed point $z$ is $R_{1}(z)$, and this is $\sim \sqrt{2 N} / \pi$ as $N \rightarrow \infty$. Rescaling at $z$ leads to the sine kernel because of the relation

$$
\lim _{N \rightarrow \infty} \frac{\pi}{\sqrt{2 N}} K_{N}\left(z+\frac{\pi x}{\sqrt{2 N}}, z+\frac{\pi y}{\sqrt{2 N}}\right)=\frac{\sin \pi(x-y)}{\pi(x-y)} .
$$

Rescaling the GUE at the "edge" of the spectrum, however, leads to a different kernel. The edge corresponds to $z \sim \sqrt{2 N}$, at which point the density is $\sim 2^{\frac{1}{2}} N^{\frac{1}{6}}$, and we have there the scaling limit [3, 11, 22

$$
\lim _{N \rightarrow \infty} \frac{1}{2^{\frac{1}{2}} N^{\frac{1}{6}}} K_{N}\left(\sqrt{2 N}+\frac{x}{2^{\frac{1}{2}} N^{\frac{1}{6}}}, \sqrt{2 N}+\frac{y}{2^{\frac{1}{2}} N^{\frac{1}{6}}}\right)=\frac{\operatorname{Ai}(x) \operatorname{Ai}^{\prime}(y)-\operatorname{Ai}^{\prime}(x) \operatorname{Ai}(y)}{x-y}
$$

where Ai is the Airy function. In previous work [28] we found several analogies between properties of this "Airy kernel" and known properties of the sine kernel: a system of partial differential equations associated with the logarithmic differential of the Fredholm determinant when the underlying domain is a union of intervals [15]; a representation of the Fredholm determinant in terms of a Painlevé transcendent in the case of a single interval [15]; and, also in this case, asymptotic expansions for these determinants and related quantities [5, 2, 29, 6, 20], achieved with the help of a differential operator which commutes with the integral operator. (See 27] for further discussion of these properties of the sine kernel.)

In this paper we show that there are completely analogous properties for a class of kernels which arise when one rescales the Laguerre or Jacobi ensembles at the edge of the spectrum. For the Laguerre ensemble the analogue of the sequence of functions $\left\{\phi_{k}(x)\right\}$ in (1.1) is obtained by orthonormalizing the sequence

$$
\left\{x^{k} x^{\alpha / 2} e^{-x / 2}\right\}
$$

over $(0, \infty)$ (here $\alpha>-1)$, whereas for Jacobi one orthonormalizes

$$
\left\{x^{k}(1-x)^{\alpha / 2}(1+x)^{\beta / 2}\right\}
$$

over $(-1,1)$. (Here $\alpha, \beta>-1$.) In the Laguerre ensemble of (positive) hermitian $N \times N$ matrices the eigenvalue density satisfies [4. 23], for a fixed $x<1$,

$$
R_{1}(4 N x) \sim \frac{1}{2 \pi} \sqrt{\frac{1-x}{x}} .
$$

This limiting law is to be contrasted with the well-known Wigner semi-circle law in the GUE. The new feature here is the "hard edge" for $x \sim 0$. At this edge we have the scaling limit [1]:

$$
\lim _{N \rightarrow \infty} \frac{1}{4 N} K_{N}\left(\frac{x}{4 N}, \frac{y}{4 N}\right)=\frac{J_{\alpha}(\sqrt{x}) \sqrt{y} J_{\alpha}^{\prime}(\sqrt{y})-\sqrt{x} J_{\alpha}^{\prime}(\sqrt{x}) J_{\alpha}(\sqrt{y})}{2(x-y)}
$$

where $J_{\alpha}(z)$ is the Bessel function of order $\alpha$. Both limits follow from the asymptotic formulas for the generalized Laguerre polynomials. (Scaling in the bulk will just lead to the sine kernel and scaling at the "soft edge," $x \sim 1$, will lead to the Airy kernel.) The same kernel arises when scaling the Jacobi ensemble at -1 or 1 . (Recall that in the Jacobi ensemble both \pm 1 are hard edges; see e.g. 23].)

For later convenience we introduce now a parameter $\lambda$ and define our "Bessel kernel" by

$$
\begin{aligned}
K(x, y): & =\lambda \frac{J_{\alpha}(\sqrt{x}) \sqrt{y} J_{\alpha}^{\prime}(\sqrt{y})-\sqrt{x} J_{\alpha}^{\prime}(\sqrt{x}) J_{\alpha}(\sqrt{y})}{2(x-y)}, \quad(x \neq y) \\
& =\frac{\lambda}{4}\left(J_{\alpha}(\sqrt{x})^{2}-J_{\alpha+1}(\sqrt{x}) J_{\alpha-1}(\sqrt{x})\right) \quad(x=y) .
\end{aligned}
$$

Before stating our results, we mention that in the cases $\alpha=\mp \frac{1}{2}$ we have, when $\lambda=1$,

$$
2 \sqrt{x y} K\left(x^{2}, y^{2}\right)=\frac{\sin (x-y)}{\pi(x-y)} \pm \frac{\sin (x+y)}{\pi(x+y)},
$$

which are kernels which arise when taking scaling limits in the bulk of the spectrum for the Gaussian orthogonal and symplectic ensembles [18]. In particular, an asymptotic expansion we derive will generalize ones found by Dyson [5] for the Fredholm determinants of these kernels.

We now state the results we have obtained. 


\section{B. The System of Partial Differential Equations}

We set

$$
J:=\bigcup_{j=1}^{m}\left(a_{2 j-1}, a_{2 j}\right) \quad\left(a_{j} \geq 0\right)
$$

and write $D(J ; \lambda)$ for the Fredholm determinant of $K$ (the operator with kernel $K(x, y)$ ) acting on $J$. If we think of this as a function of $a=\left(a_{1}, \ldots, a_{2 m}\right)$ then

$$
d \log D(J ; \lambda)=-\sum_{j=1}^{2 m}(-1)^{j} R\left(a_{j}, a_{j}\right) d a_{j}
$$

where $R(x, y)$ is the kernel of $K(I-K)^{-1}$. We introduce the notations

$$
\phi(x):=\sqrt{\lambda} J_{\alpha}(\sqrt{x}), \quad \psi(x):=x \phi^{\prime}(x)
$$

and the quantities

$$
\begin{aligned}
q_{j}:=(I-K)^{-1} \phi\left(a_{j}\right), & p_{j}:=(I-K)^{-1} \psi\left(a_{j}\right), \quad(j=1, \ldots, 2 m) \\
u:=\left(\phi,(I-K)^{-1} \phi\right), & v:=\left(\phi,(I-K)^{-1} \psi\right),
\end{aligned}
$$

where the inner products refer to the domain $J$. The differential equations are

$$
\begin{aligned}
\frac{\partial q_{j}}{\partial a_{k}} & =(-1)^{k} \frac{q_{j} p_{k}-p_{j} q_{k}}{a_{j}-a_{k}} q_{k} \quad(j \neq k) \\
\frac{\partial p_{j}}{\partial a_{k}} & =(-1)^{k} \frac{q_{j} p_{k}-p_{j} q_{k}}{a_{j}-a_{k}} p_{k} \quad(j \neq k) \\
a_{j} \frac{\partial q_{j}}{\partial a_{j}} & =p_{j}+\frac{1}{4} q_{j} u-\sum_{k \neq j}(-1)^{k} a_{k} \frac{q_{j} p_{k}-p_{j} q_{k}}{a_{j}-a_{k}} q_{k} \\
a_{j} \frac{\partial p_{j}}{\partial a_{j}} & =\frac{1}{4}\left(\alpha^{2}-a_{j}+2 v\right) q_{j}-\frac{1}{4} p_{j} u-\sum_{k \neq j}(-1)^{k} a_{k} \frac{q_{j} p_{k}-p_{j} q_{k}}{a_{j}-a_{k}} p_{k} \\
\frac{\partial u}{\partial a_{j}} & =(-1)^{j} q_{j}^{2} \\
\frac{\partial v}{\partial a_{j}} & =(-1)^{j} p_{j} q_{j} .
\end{aligned}
$$

Moreover the quantities $R\left(a_{j}, a_{j}\right)$ appearing in (1.5) are given by

$$
a_{j} R\left(a_{j}, a_{j}\right)=\sum_{k \neq j}(-1)^{k} a_{k} \frac{\left(q_{j} p_{k}-p_{j} q_{k}\right)^{2}}{a_{j}-a_{k}}+p_{j}^{2}-\frac{1}{4}\left(\alpha^{2}-a_{j}+2 v\right) q_{j}^{2}+\frac{1}{2} p_{j} q_{j} u
$$

These equations are quite similar to eqs. (1.4)-(1.9) of [28], as is their derivation.

\section{The ordinary differential equation}

For the special case $J=(0, s)$ the above equations can be used to show that $q(s ; \lambda)$, the quantity $q$ of the last section corresponding to the endpoint $s$, satisfies

$$
s\left(q^{2}-1\right)\left(s q^{\prime}\right)^{\prime}=q\left(s q^{\prime}\right)^{2}+\frac{1}{4}\left(s-\alpha^{2}\right) q+\frac{1}{4} s q^{3}\left(q^{2}-2\right) \quad\left({ }^{\prime}=\frac{d}{d s}\right) .
$$

with boundary condition 


$$
q(s ; \lambda) \sim \frac{\sqrt{\lambda}}{2^{\alpha} \Gamma(1+\alpha)} s^{\alpha / 2}, \quad s \rightarrow 0 .
$$

This equation is reducible to a special case of the $P_{V}$ differential equation; $[$ explicitly, if $q(s)=(1+y(x)) /(1-y(x))$ with $s=x^{2}$, then $y(x)$ satisfies $P_{V}$ with $\alpha^{\prime}=-\beta^{\prime}=\alpha^{2} / 8, \gamma^{\prime}=0$ and $\delta^{\prime}=-2$. (We have primed the usual $P_{V}$ parameters to avoid confusion with the $\alpha$ in our kernel. We mention that this special $P_{V}$ can be expressed algebraically in terms of a third Painlevé transcendent and its first derivative 13. We mention also that an argument can be given that (1.16) must be reducible to one of the 50 canonical types of differential equations found by Painlevé, without an explicit verification being necessary. This will be discussed at the end of section II B.) It is sometimes convenient to transform (1.16) by making the substitution

$$
q(s)=\cos \psi(s)
$$

so that $\psi$ satisfies

$$
\psi^{\prime \prime}+\frac{1}{s} \psi^{\prime}=\frac{1}{8 s} \sin (2 \psi)-\frac{\alpha^{2}}{4 s^{2}} \frac{\cos \psi}{\sin ^{3} \psi} .
$$

The Fredholm determinant is expressible in terms of $q$ by the formula

$$
D(J ; \lambda)=\exp \left(-\frac{1}{4} \int_{0}^{s} \log \frac{s}{t} q(t)^{2} d t\right) .
$$

Denoting by $R(s)$ minus the logarithmic derivative of $D(J ; \lambda)$ with respect to $s$, we have also the representation

$$
R(s)=\frac{1}{4} \cos ^{2} \psi(s)+s\left(\frac{d \psi}{d s}\right)^{2}-\frac{\alpha^{2}}{4 s} \cot ^{2} \psi(s) .
$$

Furthermore, $R(s)$ itself satisfies a differential equation which in the Jimbo-Miwa-Okamoto $\sigma$ notation for Painlevé III (see, in particular, (3.13) in [14) is

$$
\left(s \sigma^{\prime \prime}\right)^{2}+\sigma^{\prime}\left(\sigma-s \sigma^{\prime}\right)\left(4 \sigma^{\prime}-1\right)-\alpha^{2}\left(\sigma^{\prime}\right)^{2}=0
$$

where $\sigma(s)=s R(s)$; it has small $s$ expansion

$$
\begin{aligned}
\sigma(s ; \lambda)= & c_{\alpha} s^{1+\alpha}\left[1-\frac{1}{2(2+\alpha)} s+\frac{3+2 \alpha}{16(1+\alpha)(2+\alpha)(3+\alpha)} s^{2}+\cdots\right] \\
& +\frac{1}{1+\alpha} c_{\alpha}^{2} s^{2+2 \alpha}\left[1-\frac{3+2 \alpha}{2(2+\alpha)^{2}} s+\cdots\right] \\
& +\frac{1}{(1+\alpha)^{2}} c_{\alpha}^{3} s^{3+3 \alpha}[1+\cdots]+\cdots
\end{aligned}
$$

where

$$
c_{\alpha}=\frac{\lambda}{2^{2 \alpha+2}} \frac{1}{\Gamma(1+\alpha) \Gamma(2+\alpha)} .
$$

We mention that in the special case $\alpha=0$ and $\lambda=1$ we have $D(J ; 1)=e^{-s / 4}, q(s ; 1)=1, \psi(s, 1)=0$, and $\sigma(s, 1)=s / 4$ exactly [0,8,11].

\footnotetext{
${ }^{3}$ The Painlevé V differential equation is

$$
\frac{d^{2} y}{d x^{2}}=\left(\frac{1}{2 y}+\frac{1}{y-1}\right)\left(\frac{d y}{d x}\right)^{2}-\frac{1}{x} \frac{d y}{d x}+\frac{(y-1)^{2}}{x^{2}}\left(\alpha^{\prime} y+\frac{\beta^{\prime}}{y}\right)+\frac{\gamma^{\prime} y}{x}+\frac{\delta^{\prime} y(y+1)}{y-1}
$$
}

where $\alpha^{\prime}, \beta^{\prime}, \gamma^{\prime}$, and $\delta^{\prime}$ are constants. 


\section{Asymptotics}

Again we take $J=(0, s)$ and consider asymptotics as $s \rightarrow \infty$. From the random matrix point of view the interesting quantities are

$$
E(n ; s):=\left.\frac{(-1)^{n}}{n !} \frac{\partial^{n}}{\partial \lambda^{n}} D(J ; \lambda)\right|_{\lambda=1}
$$

This is the probability that exactly $n$ eigenvalues lie in $J$. The asymptotics of $E(0, s)=D(J ; 1)$ are obtained from (1.19) using the asymptotics of $q(s ; 1)$ or equivalently $\sigma(s ; 1)$ obtained from (1.16) or (1.21), respectively. (Our derivation is heuristic since as far as we are aware the corresponding Painlevé connection problem has not been rigorously solved.) We find that as $s \rightarrow \infty$,

$$
\begin{aligned}
E(0 ; s)= & \tau_{\alpha} \frac{e^{-s / 4+\alpha \sqrt{s}}}{s^{\alpha^{2} / 4}} \\
& \times\left(1+\frac{\alpha}{8} s^{-\frac{1}{2}}+\frac{9 \alpha^{2}}{128} s^{-1}+\left(\frac{3 \alpha}{128}+\frac{51 \alpha^{3}}{1024}\right) s^{-\frac{3}{2}}+\left(\frac{75 \alpha^{2}}{1024}+\frac{1275 \alpha^{4}}{32768}\right) s^{-2}+\cdots\right),
\end{aligned}
$$

where $\tau_{\alpha}$ is a constant which cannot be determined from the asymptotics of $q$ (or $\sigma$ ) alone. However, as we mentioned above, when $\alpha=\mp \frac{1}{2}$ this expansion must agree with those obtained from formulas (12.2.6) of [18] (see also (12.6.17)(12.6.19) in [18]) after replacing $s$ by $\pi^{2} t^{2}$. This leads to the conjecture

$$
\tau_{\alpha}=\frac{G(1+\alpha)}{(2 \pi)^{\alpha / 2}}
$$

where $G$ is the Barnes $G$-function [1]. This conjecture is further supported by numerical work similar to that described for the analogous conjecture in [28].

As in [28], there are two approaches to the asymptotics of $E(n ; s)$ for general $n$. We use the notation

$$
r(n ; s):=\frac{E(n ; s)}{E(0 ; s)}
$$

In the first approach (see also [2,27]) one differentiates (1.21) successively with respect to $\lambda$. Using the known asymptotics of $\sigma(s ; 1)$ and the differential equation (1.21) satisfied by $\sigma(s ; \lambda)$ for all $\lambda$, one can find asymptotic expansions for the quantities

$$
\sigma_{n}(s):=\left.\frac{\partial^{n} \sigma}{\partial \lambda^{n}}\right|_{\lambda=1}
$$

and these in turn can be used to find expansions for the $r(n ; s)$. This approach is inherently incomplete since yet another undetermined constant enters the picture. And there are also computational problems since when one expresses the $r(n, s)$ in terms of the $\sigma_{n}(s)$ a large amount of cancellation occurs, with the result that even the first-order asymptotics of $r(n ; s)$ are out of reach by this method when $n$ is large.

The second approach uses the easily-established identity

$$
r(n ; s)=\sum_{i_{1}<\ldots<i_{n}} \frac{\lambda_{i_{1}} \cdots \lambda_{i_{n}}}{\left(1-\lambda_{i_{1}}\right) \cdots\left(1-\lambda_{i_{n}}\right)}
$$

where $\lambda_{0}>\lambda_{1}>\cdots$ are the eigenvalues of the integral operator $K$ with $\lambda=1$ acting on $(0, s)$. It turns out that this operator, rescaled so that it acts on $(0,1)$, commutes with the differential operator $\mathcal{L}$ defined by

$$
\mathcal{L} f(x)=\left(x(1-x) f^{\prime}(x)\right)^{\prime}-\left(\frac{\alpha^{2}}{4 x}+\frac{s x}{4}\right) f(x),
$$

with appropriate boundary conditions on $f$. Applying the WKB method to the equation, and a simple relationship between the eigenvalues of $K$ (as functions of $s$ ) and its eigenfunctions, we are able to derive the following asymptotic formula for the eigenvalues as $s \rightarrow \infty$ :

$$
1-\lambda_{i} \sim \frac{2 \pi}{\Gamma(\alpha+i+1) i !} s^{i+\frac{\alpha+1}{2}} e^{-2 \sqrt{s}} 2^{4 i+2 \alpha+2} .
$$


From this and (1.26) we deduce

$$
r(n ; s) \sim\left\{\prod_{k=0}^{n-1} \Gamma(\alpha+k+1) k !\right\} \pi^{-n} 2^{-n(2 n+2 \alpha+1)} s^{-\frac{n^{2}}{2}-\frac{\alpha}{2} n} e^{2 n \sqrt{s}} .
$$

For the special case $\alpha=0$, the quantity $r(1 ; s)$ can be expressed exactly in terms of Bessel functions (see (2.30) below).

\section{DIFFERENTIAL EQUATIONS}

\section{A. Derivation of the system of equations}

We shall use two representations for our kernel. The first is just our definition (1.2a) using the notation (1.6),

$$
K(x, y)=\frac{\phi(x) \psi(y)-\psi(x) \phi(y)}{x-y} .
$$

The second is the integral representation

$$
K(x, y)=\frac{1}{4} \int_{0}^{1} \phi(x t) \phi(y t) d t .
$$

This follows from the differentiation formula

$$
z J_{\alpha}^{\prime}(z)=\alpha J_{\alpha}(z)-z J_{\alpha+1}(z)
$$

which gives the alternative representation

$$
\lambda \frac{\sqrt{x} J_{\alpha+1}(\sqrt{x}) J_{\alpha}(\sqrt{y})-J_{\alpha}(\sqrt{x}) \sqrt{y} J_{\alpha+1}(\sqrt{y})}{2(x-y)}
$$

for $K(x, y)$, and the Christoffel-Darboux type formula (7.14.1(9)) of [9].

Our derivation will use, several times, the commutator identity

$$
\left[L,(I-K)^{-1}\right]=(I-K)^{-1}[L, K](I-K)^{-1},
$$

which holds for arbitrary operators $K$ and $L$, and the differentiation formula

$$
\frac{d}{d a}(I-K)^{-1}=(I-K)^{-1} \frac{d K}{d a}(I-K)^{-1},
$$

which holds for an arbitrary operator depending smoothly on a parameter $a$. We shall also use the notations

$$
M=\text { multiplication by the independent variable, } \quad D=\text { differentiation, }
$$

and a subscript on an operator indicates the variable on which it acts.

It will be convenient to think of our operator $K$ as acting, not on $J$, but on $(0, \infty)$ and to have kernel

$$
K(x, y) \chi_{J}(y)
$$

where $\chi_{J}$ is the characteristic function of $J$. We continue to denote the resolvent kernel of $K$ by $R(x, y)$ and note that it is smooth in $x$ but discontinuous at $y=a_{j}$. The quantities $R\left(a_{j}, a_{j}\right)$ appearing in (1.5) are interpreted to mean

$$
\lim _{\substack{y \rightarrow a_{j} \\ y \in J}} R\left(a_{j}, y\right)
$$

and similarly for $p_{j}$ and $q_{j}$ in formulas (1.7). The definitions (1.8) of $u$ and $v$ must be modified to read

$$
u=\left(\phi \chi_{J},(I-K)^{-1} \phi\right), \quad v=\left(\phi \chi_{J},(I-K)^{-1} \psi\right),
$$


where now the inner products are taken over $(0, \infty)$. Notice that since

$$
(I-K)^{-1} \xi=(I-K)^{-1} \xi \chi_{J} \quad \text { in } J
$$

for any function $\xi$, this agrees with the original definitions $(1.8)$ of $u$ and $v$.

We have, by (2.2),

$$
\left((M D)_{x}+(M D)_{y}\right) K(x, y)=\frac{1}{4} \int_{0}^{1} t \frac{\partial}{\partial t}(\phi(x t) \phi(y t)) d t=\frac{1}{4} \phi(x) \phi(y)-K(x, y) .
$$

But it is easy to see that

$$
[M D, L] \doteq\left((M D)_{x}+(M D)_{y}+I\right) L(x, y)
$$

for any operator $L$ with kernel $L(x, y)$, where "三” means "has kernel". Taking $L(x, y)=K(x, y) \chi_{J}(y)$ gives

$$
[M D, K] \doteq \frac{1}{4} \phi(x) \phi(y) \chi_{J}(y)-\sum(-1)^{k} a_{k} K\left(x, a_{k}\right) \delta\left(y-a_{k}\right)
$$

(Recall the form (1.4) of $J$.$) It follows from this and (2.3) that$

$$
\left[M D,(I-K)^{-1}\right] \doteq \frac{1}{4} Q(x)\left(I-K^{t}\right)^{-1} \chi_{J} \phi(y)-\sum(-1)^{k} a_{k} R\left(x, a_{k}\right) \rho\left(a_{k}, y\right),
$$

where $Q(x)$, and an analogous function $P(x)$, are defined by

$$
Q(x):=(I-K)^{-1} \phi, \quad P(x):=(I-K)^{-1} \psi,
$$

where $\rho(x, y)=R(x, y)+\delta(x-y)$ is the distributional kernel of $(I-K)^{-1}$, and where $K^{t}$ is the transpose of the operator $K$. (Note that $K$ takes smooth functions to smooth functions while its transpose takes distributions to distributions.) Observe that

$$
q_{j}=Q\left(a_{j}\right), \quad p_{j}=P\left(a_{j}\right) .
$$

Next we consider commutators with $M$ and use the first representation (2.1) of $K(x, y)$. We have immediately

$$
[M, K]=(\phi(x) \psi(y)-\psi(x) \phi(y)) \chi_{J}(y),
$$

and so, by (2.3) again,

$$
\left[M,(I-K)^{-1}\right] \doteq Q(x)\left(I-K^{t}\right)^{-1} \psi \chi_{J}(y)-P(x)\left(I-K^{t}\right)^{-1} \phi \chi_{J}(y) .
$$

Notice that since

$$
\left(I-K^{t}\right)^{-1} \psi \chi_{J}=(I-K)^{-1} \psi=P \quad \text { on } J,
$$

and similarly for $\phi, Q$, we deduce

$$
R(x, y)=\frac{Q(x) P(y)-P(x) Q(y)}{x-y} \quad(x, y \in J .)
$$

In particular we have

$$
\begin{gathered}
R\left(a_{j}, a_{k}\right)=\frac{q_{j} p_{k}-p_{j} q_{k}}{a_{j}-a_{k}} \quad(j \neq k) \\
R(x, x)=Q^{\prime}(x) P(x)-P^{\prime}(x) Q(x) \quad(x \in J) .
\end{gathered}
$$

In order to compute $R\left(a_{j}, a_{j}\right)$, and also the derivatives in (1.11) and (1.12), we must find $Q^{\prime}(x)$ and $P^{\prime}(x)$. We begin with the obvious

$$
x Q^{\prime}(x)=M D(I-K)^{-1} \phi(x)=(I-K)^{-1} M D \phi(x)+\left[M D,(I-K)^{-1}\right] \phi(x) .
$$


Using (2.7), and recalling (1.6) and (2.5), we find that

$$
x Q^{\prime}(x)=P(x)+\frac{1}{4} Q(x) u-\sum(-1)^{k} a_{k} R\left(x, a_{k}\right) q_{k} .
$$

Similarly, replacing $\phi$ by $\psi$ in this derivation gives

$$
x P^{\prime}(x)=(I-K)^{-1} M D \psi(x)+\frac{1}{4} Q(x) v-\sum(-1)^{k} a_{k} R\left(x, a_{k}\right) p_{k} .
$$

To evaluate the first term on the right side we use the fact that $\phi$ satisfies the differential equation

$$
x^{2} \phi^{\prime \prime}(x)+x \phi^{\prime}(x)+\frac{1}{4}\left(x-\alpha^{2}\right) \phi(x)=0,
$$

which may be rewritten $M D \psi(x)=\frac{1}{4}\left(\alpha^{2}-x\right) \phi$. Hence

$$
\begin{aligned}
(I-K)^{-1} M D \psi(x) & =\frac{\alpha^{2}}{4} Q(x)-\frac{1}{4}(I-K)^{-1} M \phi(x) \\
& =\frac{\alpha^{2}}{4} Q(x)-\frac{x}{4} Q(x)+\frac{1}{4}\left[M,(I-K)^{-1}\right] \phi(x) .
\end{aligned}
$$

But we find, using (2.9), that

$$
\left[M,(I-K)^{-1}\right] \phi(x)=Q(x) v-P(x) u,
$$

and combining this with (2.13) and 2.15$)$ gives

$$
x P^{\prime}(x)=\frac{1}{4}\left(\alpha^{2}-x\right) Q(x)+\frac{1}{2} Q(x) v-\frac{1}{4} P(x) u-\sum(-1)^{k} a_{k} R\left(x, a_{k}\right) p_{k} .
$$

It follows from (2.11), 2.12) and (2.16) that for $x \in J$

$$
a_{j} R\left(a_{j}, a_{j}\right)=p_{j}^{2}-\frac{1}{4}\left(\alpha^{2}-a_{j}+2 v\right) q_{j}^{2}+\frac{1}{2} p_{j} q_{j} u+\sum_{k \neq j} a_{k} R\left(a_{j}, a_{k}\right)\left(q_{j} p_{k}-p_{j} q_{k}\right) .
$$

In view of (2.10) this is equation (1.15).

We now derive the differential equations (1.9)-(1.14). First, we have the easy fact that

$$
\frac{\partial}{\partial a_{k}} K \doteq(-1)^{k} K\left(x, a_{k}\right) \delta\left(y-a_{k}\right)
$$

and so by 2.4

$$
\frac{\partial}{\partial a_{k}}(I-K)^{-1} \doteq(-1)^{k} R\left(x, a_{k}\right) \rho\left(y, a_{k}\right) .
$$

At this point we use the notations $Q(x, a), P(x, a)$ for $P(x)$ and $Q(x)$ to remind ourselves that they are functions of $a$ as well as $x$. We deduce immediately from (2.17) and (2.8) that

$$
\frac{\partial}{\partial a_{k}} Q(x, a)=(-1)^{k} R\left(x, a_{k}\right) q_{k}, \quad \frac{\partial}{\partial a_{k}} P(x, a)=(-1)^{k} R\left(x, a_{k}\right) p_{k} .
$$

Since $q_{j}=Q\left(a_{j}, a\right)$ and $p_{j}=P\left(a_{j}, a\right)$ this gives

$$
\frac{\partial q_{j}}{\partial a_{k}}=(-1)^{k} R\left(a_{j}, a_{k}\right) q_{k}, \quad \frac{\partial p_{j}}{\partial a_{k}}=(-1)^{k} R\left(a_{j}, a_{k}\right) p_{k}, \quad(j \neq k) .
$$

In view of (2.10) again, these are equations (1.9) and (1.10). Moreover

$$
\frac{\partial q_{j}}{\partial a_{j}}=\left.\left(\frac{\partial}{\partial x}+\frac{\partial}{\partial a_{j}}\right) Q(x, a)\right|_{x=a_{j}}, \quad \frac{\partial p_{j}}{\partial a_{j}}=\left.\left(\frac{\partial}{\partial x}+\frac{\partial}{\partial a_{j}}\right) P\left(x, a_{j}\right)\right|_{x=a_{j}} .
$$


Equations (1.11) and (1.12) follow from this, 2.18), (2.12), 2.16) and (2.10).

Finally, using the definition of $u$ in (2.5), the fact

$$
\frac{\partial}{\partial a_{j}} \chi_{J}(y)=(-1)^{j} \delta\left(y-a_{j}\right)
$$

and (2.17) we find that

$$
\frac{\partial u}{\partial a_{j}}=(-1)^{j} \phi\left(a_{j}\right) q_{j}+(-1)^{j}\left(\phi \chi_{J}, R\left(\cdot, a_{j}\right)\right) q_{j} .
$$

But

$$
\left(\phi \chi_{J}, R\left(\cdot, a_{j}\right)\right)=\int_{J} R\left(x, a_{j}\right) \phi(x) d x=\int_{J} R\left(a_{j}, x\right) \phi(x) d x
$$

since $R(x, y)=R(y, x)$ for $x, y \in J$. Since $R(y, x)=0$ for $x \notin J$ the last integral equals

$$
\int_{0}^{\infty} R\left(a_{j}, x\right) \phi(x) d x=q_{j}-\phi\left(a_{j}\right) .
$$

This gives (1.13), and (1.14) is completely analogous.

We end this section with two relationships (analogues of (2.18) and (2.19) of [28]) which would allow us to express $u$ and $v$ in terms of the $q_{j}$ and $p_{j}$ if we wished to do so. (They will also be needed in the next section.) These are

$$
\begin{gathered}
2 v+\frac{1}{4} u^{2}+u=\sum_{j}(-1)^{j} a_{j} q_{j}^{2}, \\
u=\sum_{j}(-1)^{j}\left(4 p_{j}^{2}-\left(\alpha^{2}-a_{j}+2 v\right) q_{j}^{2}+2 p_{j} q_{j} u\right) .
\end{gathered}
$$

To obtain the first of these observe that (1.9) and (1.11) imply

$$
\left(\sum_{k} a_{k} \frac{\partial}{\partial a_{k}}\right) q_{j}=p_{j}+\frac{1}{4} q_{j} u,
$$

while from $(1.13)$ and (1.14),

$$
\frac{\partial}{\partial a_{j}}\left(2 v+\frac{1}{4} u^{2}\right)=2(-1)^{j} q_{j}\left(p_{j}+\frac{1}{4} q_{j} u\right) .
$$

If we multiply both sides of the previous formula by $(-1)^{j} a_{j} q_{j}$ and sum over $j$ what we obtain may be written

$$
\left(\sum_{k} a_{k} \frac{\partial}{\partial a_{k}}\right)\left(\sum_{j}(-1)^{j} a_{j} q_{j}^{2}\right)-\sum_{k}(-1)^{k} a_{k} q_{k}^{2}=\left(\sum_{k} a_{k} \frac{\partial}{\partial a_{k}}\right)\left(2 v+\frac{1}{4} u^{2}\right),
$$

or equivalently

$$
\left(\sum_{k} a_{k} \frac{\partial}{\partial a_{k}}\right)\left(\sum_{j}(-1)^{j} a_{j} q_{j}^{2}\right)=\left(\sum_{k} a_{k} \frac{\partial}{\partial a_{k}}\right)\left(2 v+\frac{1}{4} u^{2}+u\right) .
$$

It follows that the two sides of (2.19) differ by a function of $\left(a_{1}, \ldots, a_{2 m}\right)$ which is invariant under scalar multiplication. Since, as is easily seen, both sides vanish when all $a_{j}=0$ their difference must vanish identically.

To deduce (2.20) we multiply (2.17) by $a_{k}$ and sum over $k$ and then add the result to (2.7), recalling (2.6), to obtain

$$
\left(x \frac{\partial}{\partial x}+y \frac{\partial}{\partial y}+I+\sum_{k} a_{k} \frac{\partial}{\partial a_{k}}\right) R(x, y)=\frac{1}{4} Q(x) Q(y)
$$


for $x, y \in J$. This gives

$$
\left(\sum a_{k} \frac{\partial}{\partial a_{k}}\right) a_{j} R\left(a_{j}, a_{j}\right)=\frac{1}{4} a_{j} q_{j}^{2}=\frac{1}{4} a_{j}(-1)^{j} \frac{\partial u}{\partial a_{j}} .
$$

If we multiply both sides of this by $(-1)^{j}$ and sum over $j$ we deduce, by an argument similar to one just used, that

$$
\sum_{j}(-1)^{j} a_{j} R\left(a_{j}, a_{j}\right)=\frac{1}{4} u .
$$

Substituting for $a_{j} R\left(a_{j}, a_{j}\right)$ here the right side of (1.15) we see that the resulting double sum vanishes, and (2.20) results.

\section{B. The ordinary differential equation}

In this section we specialize to the case $J=(0, s)$ and derive (among other things) the differential equation 1.16 ) and the representation (1.19). In the notation of the last section $m=1, a_{1}=0, a_{2}=s$. We shall write $q(s), p(s), R(s)$ for $q_{2}, p_{2}, R(s, s)$, respectively. Equations (1.11)-1.14) become

$$
\begin{aligned}
s q^{\prime} & =p+\frac{1}{4} q u \\
s p^{\prime} & =\frac{1}{4}\left(\alpha^{2}-s\right) q+\frac{1}{2} q v-\frac{1}{4} p u \\
u^{\prime} & =q^{2} \\
v^{\prime} & =p q .
\end{aligned}
$$

It is immediate from (2.21) and (2.24) that

$$
(s R(s))^{\prime}=\frac{1}{4} q(s)^{2}
$$

and since

$$
\frac{d}{d s} \log D(J ; \lambda)=-R(s)
$$

(see $(1.5)$ ), we obtain the representation $(1.19)$.

To obtain the differential equation (1.16) we apply $s \frac{d}{d s}$ to both sides of (2.22) and use (2.22), (2.23) and (2.24). What results is

$$
s\left(s q^{\prime}\right)^{\prime}=\frac{1}{4}\left(\alpha^{2}-s\right) q+\frac{1}{16}\left(u^{2}+8 v\right) q+\frac{1}{4} s q^{3} .
$$

But (2.19) in this case is

$$
u^{2}+8 v=4 s q^{2}-4 u
$$

and so the above can be written

$$
s\left(s q^{\prime}\right)^{\prime}=\frac{1}{4}\left(\alpha^{2}-s\right) q-\frac{1}{4} u q+\frac{1}{2} s q^{3} .
$$

Next, we square both sides of (2.22) and use (2.20), which now says

$$
u=4 p^{2}-\left(\alpha^{2}-s+2 v\right) q^{2}+2 p q u,
$$

and find that

$$
\left(s q^{\prime}\right)^{2}=\frac{1}{4} u+\frac{1}{4}\left(\alpha^{2}-s\right) q^{2}+\frac{1}{16} q^{2}\left(u^{2}+8 v\right)
$$


Combining this with (2.27) gives

$$
s q\left(s q^{\prime}\right)^{\prime}=\left(s q^{\prime}\right)^{2}-\frac{1}{4} u+\frac{1}{4} s q^{4},
$$

and combining this with (2.28) gives the desired equation 1 1.16). The boundary condition (1.17) follows from the Neumann expansion of the defining expression (1.7) for $q$.

Using (1.18) one easily verifies that

$$
\begin{aligned}
R(s) & =\frac{1}{4} \cos ^{2} \psi+s\left(\frac{d \psi}{d s}\right)^{2}-\frac{\alpha^{2}}{4 s} \csc ^{2} \psi+\frac{c}{s} \\
& =\frac{1}{4} \cos ^{2} \psi+s\left(\frac{d \psi}{d s}\right)^{2}-\frac{\alpha^{2}}{4 s} \cot ^{2} \psi+\left(c-\frac{\alpha^{2}}{4}\right) \frac{1}{s}
\end{aligned}
$$

satisfies (2.26) where $c$ is a constant of integration. That this constant is equal to $\alpha^{2} / 4$ follows from the small $s$ expansion of $R(s)$. (Use the fact that for $s \rightarrow 0, R(s) \sim K(s, s)$ and that, as follows from (1.2b), there is no simple pole in $s$.) Equation (1.21) follows from (1.20) and (1.18).

Here is the argument why (1.16) must be reducible to some Painlevé equation (or one of the other simpler differential equations on Painlevé's list). The derivation of (1.16) used only the facts that the Bessel kernel had both forms (2.1) and (2.2) and that the function $\phi$ satisfied the differential equation (2.14). (Of course $\psi$ in (2.1) must be defined as $M D \phi$.) This equation has a 2-complex-parameter family solutions and this gives a 2-complex-parameter family of kernels defined by (2.1). They can be shown to satisfy (2.2). We replace the kernels $K(x, y)$ by $s K(s x, s y)$ and have them act on $(0,1)$ rather than $(0, s)$. These operators on $(0,1)$ depend analytically on the complex variable $s$ (except for a branch point at $s=0$ ) and the corresponding $q(s)$ can have, aside from a branch point at $s=0$, only poles which occur at the values of $s$ for which $\lambda=1$ is an eigenvalue of the operator. (The resolvent of an analytic family of compact operators has a pole whenever $\lambda=1$ is an eigenvalue.) Thus the general solution (i.e., 2-complex-parameter family of solutions) of (1.16) has only poles as moveable singularities. Since the equation is of the form $q^{\prime \prime}=$ rational function of $q^{\prime}$ and $q$, it must be reducible to one of the Painlevé types.

We mention that this argument requires $|\alpha|<1$ since it is only then that all solutions of (2.14) give compact, or even bounded, operators on $L_{2}$. For other $\alpha$ it may be that we just have to replace $L_{2}$ by an appropriate space of distributions.

$$
\text { C. } r(1 ; s) \text { for } \alpha=0
$$

If we set $\alpha=0$ and make the change of variables $s=x^{2}$, the differential equation for $\psi$ (recall (1.18)) becomes

$$
\psi^{\prime \prime}+\frac{1}{x} \psi^{\prime}=\frac{1}{2} \sin (2 \psi)
$$

and we want the solutions holomorphic at the origin. The linearization of this differential equation is the modified Bessel equation and all solutions of the linear equation are linear combinations of $I_{0}(x)$ and $K_{0}(x)$. Flaschka and Newell [10] have shown, using methods of monodromy preserving deformations and singular integral equations, that the general 2-parameter solution to (2.29) can be viewed as a "perturbation" of this linear solution. (Precisely, they derive a singular integral equation whose Neumann expansion in a particular limit gives $\psi(x)$ - the first term in this expansion is a linear combination of Bessel functions.) The one-parameter family of solutions to (2.29) that are holomorphic at the origin has the representation 10]

$$
\psi(x ; \mu)=\mu \psi_{1}(x)+\frac{\mu^{3}}{3 !} \psi_{3}(x)+\frac{\mu^{5}}{5 !} \psi_{5}(x)+\cdots
$$

where $\psi_{1}(x)=I_{0}(x)$, and $\mu^{2}=1-\lambda$. Note that we are using the slightly confusing notation $\psi(x ; \mu)$ to denote the function $\psi$ of (1.18) after the change of variables $s=x^{2}$ and $\mu^{2}=1-\lambda$. Multiple integral representations (obtained from a Neumann expansion) for the higher $\psi_{j}$ 's can be easily derived from [10].

The resolvent kernel $R(s), s=x^{2}$, is given in this special case by

$$
\begin{aligned}
4 R(s) & =\cos ^{2} \psi(x)+\left(\frac{d \psi}{d x}\right)^{2} \\
& =1+(1-\lambda)\left(\left(\psi_{1}^{\prime}\right)^{2}-\psi_{1}^{2}\right)+(1-\lambda)^{2}\left(\psi_{1}^{4}-\psi_{1} \psi_{3}+\psi_{1}^{\prime} \psi_{3}^{\prime}\right)+\cdots
\end{aligned}
$$


Thus (recall (1.23) and (1.25)

$$
r(1 ; s)=-\int_{0}^{s} R_{1}(t) d t=-2 \int_{0}^{\sqrt{s}} x R_{1}\left(x^{2}\right) d x
$$

where

$$
R_{1}(t)=\left.\frac{\partial}{\partial \lambda} R(t)\right|_{\lambda=1}
$$

Therefore

$$
\begin{aligned}
r(1 ; s) & =\frac{1}{2} \int_{0}^{\sqrt{s}} x\left(\psi_{1}^{2}(x)-\left(\psi_{1}^{\prime}(x)\right)^{2}\right) d x \\
& =\frac{1}{2} \int_{0}^{\sqrt{s}} x\left(I_{0}(x)^{2}-I_{1}^{2}(x)\right) d x \\
& =\frac{s}{2}\left(I_{0}^{2}(\sqrt{s})-\frac{1}{\sqrt{s}} I_{0}(\sqrt{s}) I_{1}(\sqrt{s})-I_{1}^{2}(\sqrt{s})\right) .
\end{aligned}
$$

The last equality follows from (5.542) of 12 .

We point out the curious fact that (after letting $\psi \rightarrow i \psi$ ) the same differential equation $(2.29)$ and closely related $\tau$-function arise in the 2D Ising model [30,21,26] except that here the boundary condition is $\psi(x) \sim \mu K_{0}(x)$ as $x \rightarrow \infty$.

\section{ASYMPTOTICS}

\section{A. Asymptotics of the $\sigma$-equation}

In the case of the finite $N$ ensemble and $\alpha=0$, Edelman [7,8] and Forrester [11] (by a direct evaluation of the integrals defining the probability $\left.E_{N}(0 ; s)\right)$ has shown that $E(0 ; s)$ is exactly equal to $e^{-s / 4}$. From this it follows, for $\alpha=0$ and $\lambda=1$, that $\sigma(s ; 1)=s / 4$. For general $\alpha$ and $\lambda=1$ it is therefore reasonable to assume an asymptotic expansion of the form:

$$
\sigma(s ; 1)=c_{1} s+c_{2} s^{1 / 2}+c_{3}+c_{4} s^{-1 / 2}+\cdots, \quad s \rightarrow \infty .
$$

Substituting this into the differential equation (1.21) results in equations that uniquely determine the coefficients $c_{j}$ once a choice in the square root $\sqrt{\alpha^{2}}$ is made. Since for $\alpha=\mp \frac{1}{2}$ our asymptotic expansion of $E(0 ; s)$ must agree with those of Dyson (recall (1.3)), we see that we must choose the square root $-\alpha-\alpha$. A calculation then gives

$$
\begin{aligned}
\sigma(s ; 1)=\frac{s}{4} & -\frac{\alpha}{2} s^{1 / 2}+\frac{\alpha^{2}}{4}+\frac{\alpha}{16} s^{-1 / 2}+\frac{\alpha^{2}}{16} s^{-1}+\frac{\alpha}{256}\left(16 \alpha^{2}+9\right) s^{-3 / 2}+\frac{\alpha^{2}}{64}\left(4 \alpha^{2}+9\right) s^{-2} \\
& +\frac{\alpha}{2048}\left(128 \alpha^{4}+720 \alpha^{2}+225\right) s^{-5 / 2}+\cdots \quad s \rightarrow \infty,
\end{aligned}
$$

from which (1.24) follows.

\section{B. Asymptotics via the commuting differential operator}

Throughout this section we take $\lambda=1$. The operator $K$, when rescaled to act on $(0,1)$ instead of $(0, s)$, has kernel $s K(s x, s y)$. By (2.2) this is equal to

$$
\frac{s}{4} \int_{0}^{1} \phi(s x t) \phi(s y t) d t
$$


and so $K$ (rescaled, as it will be throughout this section), is the square of the operator on $(0,1)$ with kernel

$$
J(x, y)=\frac{\sqrt{s}}{2} \phi(s x y)=\frac{\sqrt{s}}{2} J_{\alpha}(\sqrt{s x y}) .
$$

This function satisfies the differential equation

$$
x^{2} J_{x x}+x J_{x}+\left(\frac{s x y}{4}-\frac{\alpha^{2}}{4}\right) J=0 .
$$

The operator $J$ will commute with a differential operator

$$
\mathcal{L}=\frac{d}{d x} \alpha(x) \frac{d}{d x}+\beta(x)
$$

if $\alpha(0)=\alpha(1)=0$ and if

$$
\alpha(y) J_{y y}+\alpha^{\prime}(y) J_{y}+\beta(y) J=\alpha(x) J_{x x}+\alpha^{\prime}(x) J_{x}+\beta(x) J .
$$

If we use (3.2) we see that this will be satisfied if

$$
\begin{aligned}
& -\alpha(x)\left[x^{-1} J_{x}+\left(\frac{s y}{4 x}-\frac{\alpha^{2}}{4 x^{2}}\right)\right]+\alpha^{\prime}(x) J_{x}+\beta(x) J \\
& =\text { the same expression with } x \text { and } y \text { interchanged. }
\end{aligned}
$$

Equating the terms involving the first derivatives of $J$ gives

$$
\left(\alpha^{\prime}(x)-x^{-1} \alpha(x)\right) J_{x}=\left(\alpha^{\prime}(y)-y^{-1} \alpha(y)\right) J_{y} .
$$

But $x J_{x}=y J_{y}$, so the above will hold if

$$
x^{-1}\left(\alpha^{\prime}(x)-x^{-1} \alpha(x)\right)=y^{-1}\left(\alpha^{\prime}(y)-y^{-1} \alpha(y)\right) .
$$

This is satisfied if $\alpha(x)$ is a quadratic without constant term and, of course, we choose

$$
\alpha(x)=x(1-x) .
$$

What is required of $\beta$, then, is seen to be

$$
\beta(x)-x(1-x)\left(\frac{s y}{4 x}-\frac{\alpha^{2}}{4 x^{2}}\right)=\beta(y)-y(1-y)\left(\frac{s x}{4 y}-\frac{\alpha^{2}}{4 y^{2}}\right),
$$

which is satisfied by

$$
\beta(x)=-\frac{\alpha^{2}}{4 x}-\frac{s x}{4} .
$$

We write the differential equation, for which the eigenfunctions $f(x)$ are the eigenfunctions of $J$, as

$$
\left.\left(x(1-x) f^{\prime} x\right)\right)^{\prime}+\left(\mu \sqrt{s}-\frac{\alpha^{2}}{4 x}-\frac{s x}{4}\right) f(x)=0 .
$$

The boundary conditions are that $f(x)$ be bounded as $x \rightarrow 1$ and that $f(x)$ be asymptotic to a constant times $x^{\alpha / 2}$ as $x \rightarrow 0$. The reason we wrote the eigenvalues as we did is that for each $i$ the $\mu$ corresponding to the i'th largest eigenvalue is bounded as $s \rightarrow \infty$. This is easily seen by an oscillation argument. So we assume $i$ is fixed and proceed to find the asymptotics of the corresponding eigenfunction $f(x)$ as $s \rightarrow \infty$. We assume it normalized so that

$$
f(x) \sim x^{\alpha / 2} \text { as } x \rightarrow 0 .
$$




\section{The region $x \ll 1$}

The approximating equation is

$$
f^{\prime \prime}(x)+\frac{1}{x} f^{\prime}(x)+\left(\frac{\mu \sqrt{s}}{x}-\frac{\alpha^{2}}{4 x^{2}}-\frac{s}{4}\right) f(x)=0 .
$$

The solution of this equation which satisfies (3.4) is

$$
x^{\alpha / 2} e^{-\sqrt{s} x / 2} \Phi\left(\frac{1+\alpha}{2}-\mu, 1+\alpha, \sqrt{s} x\right)
$$

where $\Phi$ is the confluent hypergeometric function [9]. We deduce that when $x \ll 1$,

$$
f(x) \sim x^{\alpha / 2} e^{-\sqrt{s} x / 2} \Phi\left(\frac{1+\alpha}{2}-\mu, 1+\alpha, \sqrt{s} x\right) .
$$

2. The region $x \ll 1, \sqrt{s} x \gg 1$

Case 1. $\mu \neq i+\frac{1+\alpha}{2}(i=0,1, \ldots)$. Then from the known asymptotics of $\Phi$ as its argument tends to $\infty$ (6.13(3) of [9]) we deduce that

$$
f(x) \sim \frac{\Gamma(1+\alpha)}{\Gamma\left(\frac{1+\alpha}{2}-\mu\right)} s^{-\frac{1}{2}\left(\frac{1+\alpha}{2}+\mu\right)} x^{-\frac{1}{2}-\mu} e^{\sqrt{s} x / 2}
$$

Case 2. $\mu=i+\frac{1+\alpha}{2}$. Then

$$
\Phi(-i, 1+\alpha, \sqrt{s} x)=\frac{\Gamma(\alpha+1) i !}{\Gamma(\alpha+i+1)} L_{i}^{\alpha}(\sqrt{s} x)
$$

where $L_{i}^{\alpha}$ is the generalized Laguerre polynomial $(6.9(36)$ of [9]). So we find that in this case

$$
\begin{gathered}
f(x) \sim(-1)^{i} \frac{\Gamma(\alpha+1)}{\Gamma(\alpha+i+1)} s^{\frac{i}{2}} x^{i+\frac{\alpha}{2}} e^{-\sqrt{s} x / 2} . \\
\text { 3. The region } \sqrt{s} x \gg 1,(1-x) s \gg 1
\end{gathered}
$$

Here we use the standard WKB approximation for the solutions of a differential equation $\left(p f^{\prime}\right)^{\prime}+q f=0$ given by $e^{ \pm y} /(p q)^{\frac{1}{4}}$ where $y=\int \sqrt{q / p}$. In the case of our equation (3.3), the range of validity of the approximation is as indicated in the heading of this section. To be definite, we take

$$
y=-\int_{x}^{1} \sqrt{q(z) / p(z)} d z .
$$

It is easy to compute that for the range in question we have

$$
y=-\sqrt{s} \sqrt{1-x}-\mu \log x+2 \mu \log (1+\sqrt{1-x})+o(1) .
$$

Also, in this case $p q$ is asymptotically $-s / 4$ times $x^{2}(1-x)$. Hence

$$
\begin{aligned}
& f(x) \sim\left(a(s) x^{-\mu}(1+\sqrt{1-x})^{2 \mu} e^{-\sqrt{s} \sqrt{1-x}}\right. \\
& \left.+b(s) x^{\mu}(1+\sqrt{1-x})^{-2 \mu} e^{\sqrt{s} \sqrt{1-x}}\right)
\end{aligned}
$$

where $a(s)$ and $b(s)$ are constants depending on $s$. 


\section{The region $x \rightarrow 1$}

Letting $y=1-x, f(x)=g(y)$ gives the approximating equation

$$
\left(y g(y)^{\prime}\right)^{\prime}-\frac{s}{4} g(y)=0 .
$$

The general solution of this is a constant times $I_{0}(\sqrt{y})$ where $I_{0}$ is the modified Bessel function. Thus we deduce that as $x \rightarrow 1$

$$
f(x) \sim c(s) I_{0}(\sqrt{s(1-x)})
$$

for some $\mathrm{c}(\mathrm{s})$.

\section{Determination of $a(s), b(s)$ and $c(s)$}

From the asymptotics of $I_{0}$ at infinity and (3.10) we deduce that

$$
f(x) \sim \frac{c(s)}{\sqrt{2 \pi} s^{\frac{1}{4}}} \frac{e^{\sqrt{s} \sqrt{1-x}}}{(1-x)^{\frac{1}{4}}}
$$

when $x \rightarrow 1$ and $s(1-x) \rightarrow \infty$. Comparing this with (3.9) shows that $a(s)=0$ and that

$$
c(s) \sim \sqrt{2 \pi} s^{\frac{1}{4}} b(s) .
$$

And now comparing (3.9) with (3.6) and (3.8) in their overlapping range of validity we see that we must be in case 2 and that

$$
b(s) \sim(-1)^{i} \frac{\Gamma(\alpha+1)}{\Gamma(\alpha+i+1)} 2^{2 n+\alpha+1} e^{-\sqrt{s}} .
$$

What we shall need from all this is, first, the asymptotics of $f(1)$. This follows immediately from (3.10), (3.11), (3.12), and the fact that $I_{0}(0)=1$ :

$$
f(1) \sim(-1)^{n} \sqrt{2 \pi} \frac{\Gamma(\alpha+1)}{\Gamma(\alpha+i+1)} s^{\frac{i}{2}+\frac{1}{4}} e^{-\sqrt{s}} 2^{2 n+\alpha+1} .
$$

We shall also need the asymptotics of $\int f(x)^{2} d x$. It follows from the asymptotics we have derived that the main contribution to this integral comes from an arbitrarily small neighborhood of $x=0$. It follows from (3.5), (3.7) and the fact

$$
\int_{0}^{\infty} x^{\alpha} e^{-x} L_{i}^{\alpha}(x)^{2} d x=\frac{\Gamma(\alpha+i+1)}{i !}
$$

that

$$
\int_{0}^{1} f(x)^{2} d x \sim \frac{\Gamma(\alpha+1)^{2} i !}{\Gamma(\alpha+i+1} s^{-\frac{\alpha}{2}-\frac{1}{2}} .
$$

We put these two relations together to get what we really want, which is

$$
\frac{f(1)^{2}}{\int_{0}^{1} f(x)^{2} d x} \sim \frac{2 \pi}{\Gamma(\alpha+i+1) i !} s^{i+\frac{\alpha}{2}+1} e^{-2 \sqrt{s}} 2^{4 i+2 \alpha+2} .
$$




\section{The asymptotics of $\lambda_{i}$}

Since $\mathcal{L}$ commutes with our integral operator $K$, rescaled to act on $(0,1)$, the set $\left\{f_{i}\right\}$ of eigenfunctions corresponding to the eigenvalues $\mu_{0}<\mu_{1}<\ldots$ of $\mathcal{L}$ is the set of eigenfunctions of $K$ corresponding to its eigenvalues, in some order.

Lemma 1. The eigenvalues of $K$ are simple.

Proof. Since the eigenvalues of $K$ are the squares of the eigenvalues of $J$ what we have to show is that if $f_{1}(x)$ and $f_{2}(x)$ are eigenfunctions of $\mathcal{L}$ (they need not correspond to any particular $\mu$ here) and if for some $\nu$ we have either

$$
\int_{0}^{1} J_{\alpha}(\sqrt{s x y}) f_{i}(y) d y=\nu f_{i}(x) \quad(i=1,2)
$$

or

$$
\int_{0}^{1} J_{\alpha}(\sqrt{s x y}) f_{1}(y) d y=\nu f_{1}(x), \quad \int_{0}^{1} J_{\alpha}(\sqrt{s x y}) f_{2}(y) d y=-\nu f_{2}(x)
$$

then $f_{1}$ and $f_{2}$ are linearly dependent. We shall assume them normalized so that they both have the value 1 at $x=1$.

Notice first that $\nu \neq 0$ for otherwise if we expand the $f_{1}$ equation near $x=0$ we would find that

$$
\int_{0}^{1} x^{\frac{\alpha}{2}+k} f_{1}(x) d x=0, \quad k=0,1, \ldots
$$

and $f_{1}$ would be identically 0 .

Next we define

$$
H(x)=e^{-x / 2} J_{\alpha}\left(\sqrt{s e^{-x}}\right), g_{i}(x)=e^{-x / 2} f_{i}\left(e^{-x}\right),
$$

make the obvious variable changes, and find that our relations become

$$
\begin{gathered}
\int_{0}^{\infty} H(x+y) g_{i}(y) d y=\nu g_{i}(x), \quad(i=1,2), \\
\int_{0}^{\infty} H(x+y) g_{1}(y) d y=\nu g_{1}(x), \quad \int_{0}^{\infty} H(x+y) g_{2}(y) d y=-\nu g_{2}(x) .
\end{gathered}
$$

What comes now is almost identical to the proof of Lemma 1 of [28]. Assuming first that (3.15) holds, we differentiate twice this relation with $i=1$ and then integrate by parts twice to obtain

$$
\nu g_{1}(x)=-H^{\prime}(x)+H(x) g_{1}^{\prime}(0)+\int_{0}^{\infty} H(x+y) g_{1}(y) d y .
$$

If we multiply both sides by $g_{2}(x)$ and integrate, using (3.15) and its differentiated version, we obtain (recall that $\left.g_{i}(0)=1\right)$

$$
\nu \int_{0}^{\infty} g_{1}^{\prime \prime}(x) g_{2}(x) d x=-\nu g_{2}^{\prime}(0)+\nu g_{1}^{\prime}(0)+\nu \int_{0}^{\infty} g_{2}(y) g_{1}^{\prime \prime}(y) d y .
$$

Thus, since $\nu \neq 0$, we have $g_{1}^{\prime}(0)=g_{2}^{\prime}(0)$. Equivalently, $f_{1}^{\prime}(1)=f_{2}^{\prime}(1)$. But since also $f_{1}(1)=f_{2}(1)$, it follows from equation (2) that the corresponding eigenvalues $\mu$ must be the same, and so the eigenfunctions are the same.

Next, assume (3.16) holds. Differentiating both sides of the first relation once and integrating by parts give

$$
\nu g_{1}^{\prime}(x)=-H(x)-\int_{0}^{\infty} H(x+y) g_{1}^{\prime}(y) d y
$$

Multiplying both sides of this by $g_{2}(x)$ and integrating, using the second part of (3.16), we obtain

$$
\nu \int_{0}^{\infty} g_{1}^{\prime}(x) g_{2}(x) d x=-\nu+\nu \int_{0}^{\infty} g_{2}(y) g_{1}^{\prime}(y) d y
$$


contradicting $\nu \neq 0$.

Now that we know the eigenvalues $\lambda_{i}$ of $K$ are simple we can order them so that $\lambda_{0}>\lambda_{1}>\ldots$. There is a permutation $\sigma$ of $\mathbf{N}$ such that the eigenvalue corresponding to $f_{i}$ is $\lambda_{\sigma(i)}$. This permutation is independent of $s$ since the eigenvalues $\mu_{i}$ and $\lambda_{i}$ both vary continuously with $s$. The next lemma allows us to compute $d \lambda_{i} / d s$ for each $i$.

Lemma 2. Let $\lambda$ be a simple eigenvalue of an integral operator on $(0,1)$ with symmetric kernel of the form $s K(s x, s y)$ where $K(x, y)$ is smooth and symmetric. Let $f$ be a corresponding eigenfunction. Then

$$
\frac{d \lambda}{d s}=\frac{\lambda}{s} \frac{f(1)^{2}}{\int_{0}^{1} f(x)^{2} d x} .
$$

Proof. We may assume $f$ normalized so that $\int_{0}^{1} f(x)^{2} d x=1$. Then according to Lemma 3 of [28] we have

$$
\frac{d \lambda}{d s}=\int_{0}^{1} \int_{0}^{1} \frac{\partial}{\partial s}(s K(s x, s y)) f(y) f(x) d y d x .
$$

(This holds no matter what the form of the kernel.) In the case at hand the first factor in the integrand equals

$$
K(s x, s y)+\left(x \frac{\partial}{\partial x}+y \frac{\partial}{\partial y}\right) K(s x, s y)
$$

and we easily deduce from this that the integral itself equals

$$
\frac{\lambda}{s}\left(\int_{0}^{1} f(x)^{2} d x+\int_{0}^{1} x f^{\prime}(x) f(x) d x+\int_{0}^{1} y f^{\prime}(y) f(y) d y\right)
$$

and integration by parts shows that the expression in parentheses equals $f(1)^{2}$.

Remark. It is easily seen that the conclusion of the lemma holds when the kernel has a mild singularity at 0 such as our Besssel kernel has when $\alpha<0$.

Lemma 3. For each $i$ we have $\lambda_{i} \rightarrow 1$ as $s \rightarrow \infty$.

Proof. The Hankel transform, when rescaled by the variable change $x \mapsto \sqrt{x}$, is the integral operator $H$ on $(0, \infty)$ with kernel $\frac{1}{2} J_{\alpha}(\sqrt{x y})$ and so our operator $K$ on $(0, s)$ may be thought of as $P_{s} H P_{s} H P_{s}$ where $P_{s}$ denotes the projection from $L_{2}(0, \infty)$ to $L_{2}(0, s)$. Since, as is well-known, $H^{2}=I$, the minimax characterization of the eigenvalues shows that for each $i$ the eigenvalue of $P_{s} H P_{s}$ with i'th largest absolute value tends to \pm 1 as $s \rightarrow \infty$. Since our operator is the square of this one, the statement of the lemma follows.

We can now deduce the asymptotic formula (15) for the eigenvalues $\lambda_{i}$. We apply Lemma 2 to the eigenvalue $\lambda_{\sigma(i)}$ associated with the eigenfunction $f_{i}(x)$ of (3.3) and use (3.14) to deduce

$$
\frac{d \log \lambda_{\sigma(i)}}{d s} \sim \frac{2 \pi}{\Gamma(\alpha+i+1) i !} s^{i+\frac{\alpha}{2}} e^{-2 \sqrt{s}} 2^{4 i+2 \alpha+2} .
$$

Recalling Lemma 3 we see that we can integrate from $s$ to $\infty$ and we obtain

$$
1-\lambda_{\sigma(i)} \sim \frac{2 \pi}{\Gamma(\alpha+i+1) i !} s^{i+\frac{\alpha+1}{2}} e^{-2 \sqrt{s}} 2^{4 i+2 \alpha+2} .
$$

It remains to show that $\sigma(i)=i$ for all $i$. But it is clear from the above formula that $i<j$ implies that $\lambda_{\sigma(i)}>\lambda_{\sigma(j)}$ for large $s$ (and so for all $s$ ) and therefore $\sigma(i)<\sigma(j)$. Since $\sigma: \mathbf{N} \rightarrow \mathbf{N}$ is onto, we must have $\sigma(i)=i$ for all $i$.

\section{ACKNOWLEDGMENTS}

We wish to thank Professor F. J. Dyson for bringing to our attention the notion of "hard edge" random matrix ensembles. This work was supported in part by the National Science Foundation, DMS-9001794 and DMS-9216203, and this support is gratefully acknowledged. 
[1] E. W. Barnes, The theory of the G-function, Quart. J. Pure and Appl. Math. 31 (1900) 264-314.

[2] E. L. Basor, C. A. Tracy, and H. Widom, Asymptotics of level spacing distributions for random matrices, Phys. Rev. Letts. 69 (1992) 5-8.

[3] M. J. Bowick and E. Brézin, Universal scaling of the tail of the density of eigenvalues in random matrix models, Phys. Letts. B268 (1991) 21-28.

[4] B. V. Bronk, Exponential ensemble for random matrices, J. Math. Phys. 6 (1965) 228-237.

[5] F. J. Dyson, Fredholm determinants and inverse scattering problems, Commun. Math. Phys. 47 (1976) $171-183$.

[6] F. J. Dyson, The Coulomb fluid and the fifth Painlevé transcendent, IASSNSS-HEP-92/43 preprint, to appear in the proceedings of a conference in honor of C. N. Yang, ed. S.-T. Yau.

[7] A. Edelman, Eigenvalues and Condition Numbers of Random Matrices, SIAM J. Matrix Anal. Appl. 9 (1988) 543-560.

[8] A. Edelman, The distribution and moments of the smallest eigenvalue of a random matrix of Wishart type, Linear Alg. and Its Appl. 159 (1991) 55-80.

[9] A. Erdélyi (ed.), Higher Transcendental Functions, Vols. I and II (McGraw-Hill, New York, 1953).

[10] H. Flaschka and A. C. Newell, Monodromy- and Spectrum-Preserving Deformations I, Commun. Math. Phys. 76 (1980) 65-116.

[11] P. J. Forrester, The spectrum edge of random matrix ensembles, preprint.

[12] I. S. Gradshteyn and I. M. Ryzhik, Tables of Integrals, Series, and Products, Corrected and Enlarged Edition (Academic, San Diego, 1980).

[13] V. I. Gromak, Reducibility of Painlevé equations, Diff. Urav. 20 (1984) 1674-1683.

[14] M. Jimbo, Monodromy problem and the boundary condition for some Painlevé equations, Publ. RIMS, Kyoto Univ. 18 (1982) 1137-1161.

[15] M. Jimbo, T. Miwa, Y. Môri, and M. Sato, Density matrix of an impenetrable Bose gas and the fifth Painlevé transcendent, Physica 1D (1980) 80-158.

[16] R. D. Kamien, H. D. Politzer, and M. B. Wise, Universality of random-matrix predictions for the statistics of energy levels, Phys. Rev. Letts. 60 (1988) 1995-1998.

[17] G. Mahoux and M. L. Mehta, A method of integration over matrix variables: IV, J. Phys. I France 1 (1991) $1093-1108$.

[18] M. L. Mehta, Random Matrices, 2nd edition (Academic, San Diego, 1991).

[19] M. L. Mehta, A non-linear differential equation and a Fredholm determinant, J. de Phys. I France, 2 (1992) $1721-1729$.

[20] M. L. Mehta and G. Mahoux, Level spacing functions and non-linear differential equations, to appear in J. Phys. I France.

[21] B. M. McCoy, C. A. Tracy, and T. T. Wu, Painlevé functions of the third kind, 18 (1977) $1058-1092$.

[22] G. Moore, Matrix models of 2D gravity and isomonodromic deformation, Prog. Theor. Physics Suppl. No. 102 (1990) 255-285.

[23] T. Nagao and M. Wadati, Correlation functions of random matrix ensembles related to classical orthogonal polynomials, J. Phys. Soc. Japan 60 (1991) 3298-3322.

[24] L. A. Pastur, On the universality of the level spacing distribution for some ensembles of random matrices, Letts. Math. Phys. 25 (1992) 259-265.

[25] C. E. Porter, Statistical Theory of Spectra: Fluctuations (Academic, New York, 1965).

[26] M. Sato, T. Miwa, and M. Jimbo, Holonomic quantum fields, III, IV, Publ. RIMS Kyoto Univ. 15 (1979) 577-629; 15 (1979) 871-972.

[27] C. A. Tracy and H. Widom, Introduction to random matrices, to appear in the proceedings of the $8^{\text {th }}$ Scheveningen Conference, Springer Lecture Notes in Physics.

[28] C. A. Tracy and H. Widom, Level spacing distributions and the Airy kernel, to appear in Commun. Math. Phys.

[29] H. Widom, The asymptotics of a continuous analogue of orthogonal polynomials, to appear in J. Approx. Th.

[30] T. T. Wu, B. M. McCoy, C. A. Tracy, and E. Barouch, Spin-spin correlation functions for the two-dimensional Ising model: Exact theory in the scaling region, Phys. Rev. B13 (1976) 316-374. 